\title{
Modified spectral-domain approach for microstrip lines with finite metallisation thickness and conductivity
}

\author{
J.-Y. Ke \\ C.H. Chen
}

Indexing terms: Microstrip line, Finite metallisation thickness, Spectral-domain approach, Phase constant, Attenuation constant

\begin{abstract}
A modified spectral-domain approach is proposed for an analysis of the microstrip line whose signal strip and ground plane have finite thickness and conductivity. To improve the accuracy of the results, all three components of the strip current which have three-dimensional dependence are included in the analysis. With the basis functions properly chosen, this new approach can be treated as easily as the conventional spectraldomain approach. By this modified approach, both the phase constant and attenuation constant can be determined simultaneously without using the assumption that the skin depth is much larger or smaller than the signal strip thickness. In this work, comparison with published theoretical and experimental results is presented to check the accuracy of the new approach. In particular, the effective dielectric constant and attenuation constant of a microstrip line with finite metallisation thickness and finite conductivity are discussed in detail, together with the longitudinal current distributions along the signal strip.
\end{abstract}

\section{Introduction}

Previous analysis of microwave planar wave-guiding structures was usually conducted under the assumptions of infinitely thin strip and infinite conductivity. Recently, the problem of finite metallisation thickness and finite conductivity has received increased attention, because strip thickness may be comparable to skin depth in practical monolithic microwave integrated circuits (MMICs). With metallisation thickness in the order of skin depth, the propagation characteristics, especially the attenuation constant, would behave different from previous ones which assume zero metallisation thickness or infinite conductivity but with finite metallisation thickness. Thus, a more reliable and accurate conductor-loss model is needed in the design of monolithic microwave and millimetre-wave integrated circuits [1].

To deal with the effect of finite metallisation thickness but with infinite conductivity, full-wave approaches such as the variational conformal mapping technique [2], frequency-dependent hybrid-mode formulation [3],

\section{(C) IEE, 1995}

Paper 1908H (E12), received 10th November 1994

The authors are with the Department of Electrical Engineering, National Taiwan University, Taipei, Taiwan 10617, Republic of China

IEE Proc.-Microw. Antennas Propag., Vol. 142, No, 4, August 1995 boundary integral equation method $[4,5]$ and spectral domain method [6] have been proposed. However, these methods can obtain only the effective dielectric constant.

To discuss the conductor loss of microstrip lines by the conventional power-loss method [7], the signal strip thickness was assumed to be much larger than the skin depth (called the skin-depth approximation) and the currents were evaluated from the structure with zero metallisation thickness [8-11]. Recently, some full-wave approaches, such as the transverse resonance technique [12] and the extended spectral domain approach [1315], were proposed to deal with the problem of finite strip thickness and finite conductivity. In these methods, the conductivity was first regarded as infinite to obtain the effective dielectric constant and the fields, and then these fields and the power-loss method were used to determine the loss caused by the imperfect conductor of the signal strip.

Also by using the skin-depth approximation, some investigators [16-18] have treated the finite-thickness lossy strip as a surface impedance boundary condition by which the effective dielectric constant and the attenuation constant can be determined simultaneously. Again, in [19], the microstrip conductor loss was evaluated by representing the lossy signal strip by an equivalent impedance surface; however, this surface was characterised by a frequency-dependent surface impedance which was derived from a quasi-TEM analysis of the fields and currents inside the imperfectly conducting strip. Recently, full-wave techniques without using the skin-depth approximation, such as the mode-matching method [20], method of lines [21], and integral equation method [22] were proposed to give a better characterisation of both effective dielectric constant and attenuation constant.

The power-loss method and the methods based on the skin-depth approximation are not suitable for cases in which the skin depth and the strip thickness are of the same order. Also, the above-mentioned full-wave techniques are either time consuming or lack generality due to some assumptions made. Therefore, an alternative method having fewer assumptions to obtain the propagation constant is needed to meet the development of MMIC technology.

For an analysis of the microstrip line with layer structure in which the thickness and conductivity of the signal

This work was supported by the National Science Council of the Republic of China under Grant NSC 82-0404-E002-095. 
strip and ground plane are finite, a new formulation modifying the conventional spectral-domain approach is proposed to handle the case with three-dimensional dependence in current distributions. In this study, all three components of strip current are included to improve the accuracy and the effects of substrate and ground plane are discussed. Also by this modification, the integration along one coordinate variable is analytically integrated. Hence, it is as easy as the conventional spectral-domain approach in which only one-dimensional integration has to be performed to obtain the matrix equation for the propagation constant

\section{Formulation}

The cross-section of the microstrip line is shown in Fig. $1 a$. The conductivity and thickness of the signal strip are
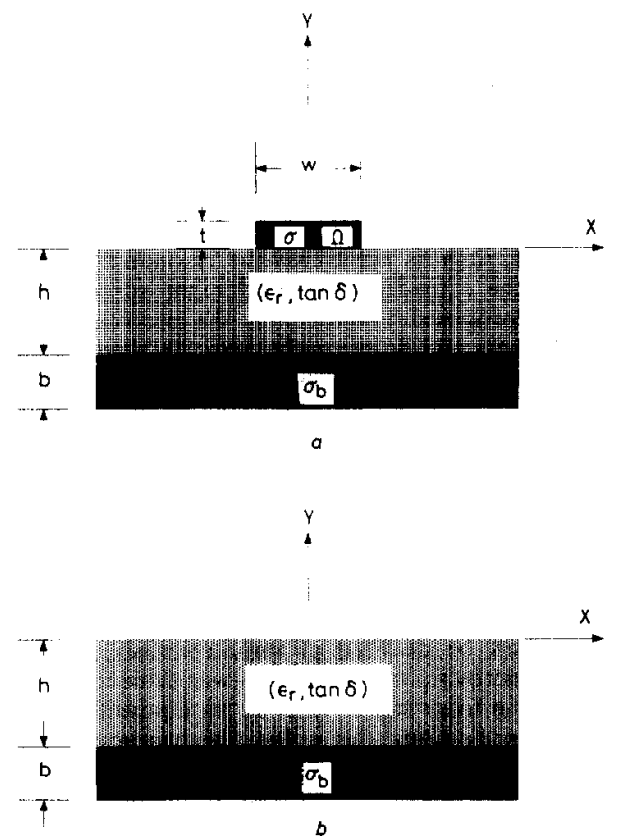

Fig. 1 (a) Cross section of microstrip line and (b) the layer structure for deriging Green's functions

$\sigma$ and $t$, and those of the ground plane are $\sigma_{b}$ and $b$. The width of the signal strip is $w$; and the thickness, dielectric constant and loss tangent of the substrate are $h, \varepsilon_{r}$ and $\tan \delta$, respectively. The relation between the electric field $E(r)$ and the current density $J(r)$ within the strip conductor $\Omega$ is

$$
E(r)=\int_{\mathbf{\Omega}} \boldsymbol{G}\left(\boldsymbol{r}-\boldsymbol{r}^{\prime}\right) \cdot J\left(\boldsymbol{r}^{\prime}\right) d \boldsymbol{r}^{\prime}=\frac{1}{\sigma} J(\boldsymbol{r})
$$

Here, $\boldsymbol{G}$ is the dyadic Green's function for the layer structure (Fig. $1 b$ ) in which the signal strip is replaced by the free space. It should be emphasised that the ground plane is now regarded as a lossy layer with parameters $\sigma_{b}$ and $b$, therefore the effect of lossy ground may be included and discussed by these Green's functions. Some detail of the Green functions is presented in the Appendix.

All field quantities are assumed to be of the form $\exp \left[j\left(\omega t-k_{z} z\right)\right]$, and the Fourier transformation pair is defined as

$$
\begin{aligned}
\widetilde{A}\left(k_{x}\right) & =\int_{-\infty}^{\infty} A(x) e^{-j k_{x} x} d x \\
A(x) & =\frac{1}{2 \pi} \int_{-\infty}^{\infty} \tilde{A}\left(k_{x}\right) e^{j k_{x} x} d k_{x}
\end{aligned}
$$

By weighting both sides of eqn. 1 by any arbitrary function $\boldsymbol{w}(\boldsymbol{r})$ and then integrated, one may obtain the integral equation

$$
\begin{array}{r}
\int_{-w / 2}^{w / 2} \int_{0}^{t} \omega(x, y) \cdot\left[\int_{-w / 2}^{w / 2} \int_{0}^{t} G\left(x, y, x^{\prime}, y^{\prime}, k_{z}\right)\right. \\
\left.\cdot J\left(x^{\prime}, y^{\prime}\right) d y^{\prime} d x^{\prime}-\frac{1}{\sigma} J(x, y)\right] d y d x=0
\end{array}
$$

It is interesting to note that the $y$-dependence form of the spectral-domain Green functions is a linear combination of $\exp \left(j \beta_{0} y\right)$ and $\exp \left(j \beta_{0} y^{\prime}\right)$, where $\beta_{0}$ is independent of $y$ or $y^{\prime}$ (see Appendix). Thus, if the bases of $J(x, y)$ are properly chosen, the integral equation eqn. 3 can be significantly simplified.

With the parameters $\left(b, \sigma_{b}\right)$ of the ground plane absorbed in the Green's functions, the only unknowns are the current distributions within the signal strip, which can be represented by

$$
\begin{aligned}
& J_{z}(x, y)=\sum_{i=0}^{m} \sum_{j=0}^{n} a_{i j} \psi_{z}^{i}(x) \phi_{z}^{j}(y) \\
& J_{x}(x, y)=\sum_{i=0}^{m} \sum_{j=0}^{n} b_{i j} \psi_{x}^{i}(x) \phi_{x}^{j}(y) \\
& J_{y}(x, y)=\sum_{i=0}^{m} \sum_{j=0}^{n} c_{i j} \psi_{y}^{i}(x) \phi_{y}^{j}(y)
\end{aligned}
$$

Here $(m+1) x$-dependent and $(n+1) y$-dependent bases are included in the approximation of the strip currents. It should be pointed out that to obtain more accurate results, all three components of strip current should be included in the analysis.

Because the strip thickness and conductivity are finite, the current distributions near the strip corner must be finite. In this study, Legendre polynomials are chosen as the $x$-dependent bases for the unknown strip current $J$, that is

$$
\begin{aligned}
& \psi_{z}^{l}(x)=\psi_{y}^{l}(x)=P_{2 l}\left(\frac{x}{w / 2}\right) \\
& \psi_{x}^{l}(x)=P_{21+1}\left(\frac{x}{w / 2}\right)
\end{aligned}
$$

For simplicity, the following piecewise linear functions will be chosen as the $y$-dependent bases for the unknown current $\boldsymbol{J}$ :

$$
\begin{aligned}
\phi_{z}^{l}(y)= & \phi_{x}^{l}(y)=\phi_{y}^{l}(y)=\Lambda(y) \\
= & \begin{cases}\frac{y-\Delta_{l-1}}{\Delta} & \Delta_{l+1}<y<\Delta_{l} \\
\frac{\Delta_{l+1}-y}{\Delta} & \Delta_{l}<y<\Delta_{l+1} \\
0 & \text { otherwise }\end{cases}
\end{aligned}
$$

where $\Delta_{t}=l \Delta$ and $\Delta=t / n$. The piecewise linear functions are chosen so that the $y$-dependent integrations may be analytically integrated. As the frequency increases, the skin depth $\delta$ decreases and the current distributions

IEE Proc.-Microw. Antennas Propag., Vol. 142, No. 4, August 1995 
along the $y$-direction tend to the exponential function $\exp (-(1+j) y / \delta)$, especially when $t>3 \delta$, and $w \gg \delta$. Due to this exponential decay behaviour in currents, we alternatively use the following exponential functions for the $y$-dependent bases in the higher frequency regime:

$$
\phi_{z}^{l}(y)=\phi_{y}^{l}(y)=\phi_{x}^{l}(y)=\left\{\begin{array}{l}
\exp \left[-\frac{1+j}{\delta} l y\right] \\
\exp \left[-\frac{1+j}{\delta} l(t-y)\right]
\end{array}\right.
$$

where eqn. $7 a$ and eqn. $7 b$ specify the current distributions over the lower and upper sides of the signal strip, respectively. Specifically, when $l=1$ the term in eqn. $7 a$ will exhibit the current distribution over the signal strip if the width $w$ approaches infinity. The completeness of the bases in eqn. 7 can be visualised by the substitutions:

$$
Y_{l}=\exp \left[-\frac{1+j}{\delta} y\right] \text { and } Y_{u}=\exp \left[-\frac{1+j}{\delta}(t-y)\right]
$$

The set (eqn. 7) is complete because any current distribution can be expressed as the polynomials of $Y_{l}$ and $Y_{u}$

To derive the matrix equation for the propagation constant, Galerkin's method is used, in which the bases for $w(x, y)$ are the same as those for $J(x, y)$. By applying the Fourier transformation and Parseval's theorem with respect to $x$ variable to eqn. 3 , then analytically integrating it with respect to $y$, one may obtain the governing equations in the spectral domain

$$
\begin{aligned}
& \sum_{i=0}^{m} \sum_{j=0}^{n} \int_{-\infty}^{\infty} \tilde{\psi}_{p}^{u}\left(k_{x}\right)\left[a_{i j} Z_{p z}^{j v}\left(k_{x}, k_{z}\right) \tilde{\psi}_{z}^{i}\left(k_{x}\right)\right. \\
& \quad+b_{i j} Z_{p x}^{j v}\left(k_{x}, k_{z}\right) \tilde{\psi}_{x}^{i}\left(k_{x}\right)+c_{i j} Z_{p y}^{j v}\left(k_{x}, k_{z}\right) \tilde{\psi}_{y}^{i}\left(k_{x}\right) \\
& \left.\quad-a_{i j} P_{p}^{j v} \tilde{\psi}_{p}^{i}\left(k_{x}\right)\right] d k_{x}=0
\end{aligned}
$$

where

$$
\begin{aligned}
& Z_{p q}^{j o}\left(k_{x}, k_{z}\right)=\int_{0}^{t} \int_{0}^{t} \tilde{G}_{p q}\left(k_{x}, k_{z} ; y, y^{\prime}\right) \phi_{q}^{j}\left(y^{\prime}\right) \phi_{p}^{v}(y) d y^{\prime} d y \\
& P_{p}^{j v}=\int_{0}^{t} \frac{1}{\sigma} \phi_{p}^{j}(y) \phi_{p}^{v}(y) d y, \quad p, q \in\{x, y, z\}
\end{aligned}
$$

Finally from eqn. 8 , one may establish the homogeneous matrix equation for determining the propagation constant $k_{x}=\beta-j \alpha$.

Note that only single integration with respect to $k_{x}$ is involved in the final spectral-domain equations (eqn. 8), since the $y$-dependent integrations in eqn. 9 have been analytically integrated. Thus, it can use the conventional technique of spectral-domain approach to find the phase and attenuation constants.

\section{Numerical results}

Numerical results such as effective dielectric constant $\varepsilon_{\text {eff }}=\beta^{2} / k_{0}^{2}\left(k_{0}^{2}=\omega^{2} \mu_{0} \varepsilon_{0}\right)$, attenuation constant $\alpha$, and longitudinal current distributions $J_{z}$ over the signal strip are investigated in detail.

The convergence behaviour of the results is depicted in Fig. 2 in which the effective dielectric constant and attenuation constant at $7 \mathrm{GHz}$ are plotted against the number of $x$ - and $y$-dependent bases. Here, the $k_{x}$ integral is calculated numerically from zero to $60 \pi / \omega$ along the real axis. In this comparison, the piecewise linear functions (eqn. 6) are adopted as the $y$-dependent bases for the strip current. As shown in Fig. 2, the effective dielectric constant converges relatively quickly when compared to the attenuation constant. In this special case, the

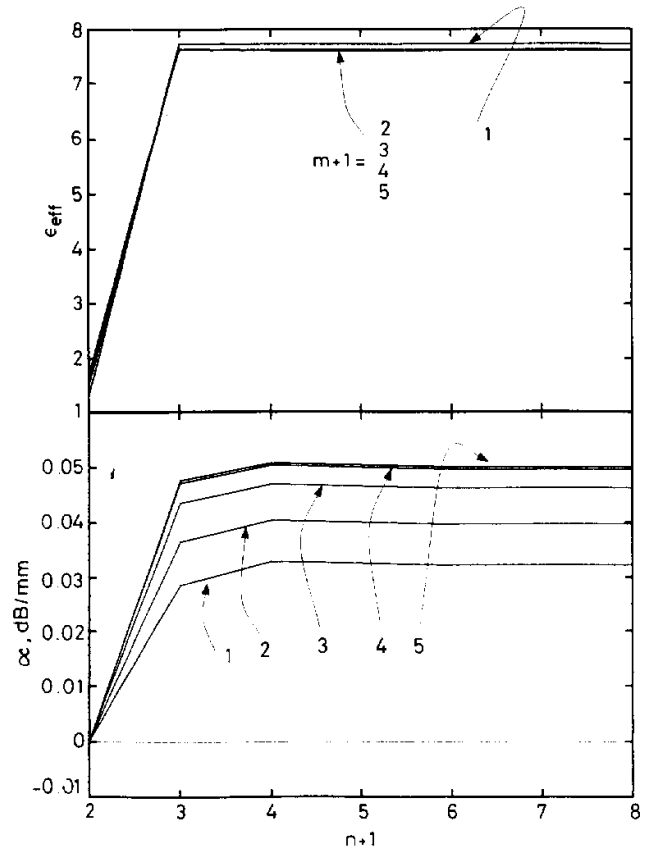

Fig. 2 Convergence of effective dielectric constant $\varepsilon_{\text {eff }}$ and attenuation constant $\alpha$ with respect to the number of $x$ - and $y$-dependent bases frequency $=7 \mathrm{GHz}, h=0.1 \mathrm{~mm}, \quad \varepsilon_{r}=12.9, \quad \tan \delta=3 \times 10^{-4}, \quad w=20 \mu \mathrm{m}$, $=3 \mu \mathrm{m}, b=12 \mu \mathrm{m}, a=\sigma_{b}=4.1 \times 10^{7} \mathrm{~S} / \mathrm{m}$

effective dielectric constant needs only two $x$-dependent bases and three $y$-dependent bases whereas the attenuation constant requires four $x$ - and $y$-dependent bases for good convergence. In comparison with the conventional spectral-domain approach which needs two bases for each component of surface current, this new method can solve the two-dimensional-dependence current distributions and obtain the equations as easily as the conventional ones at the cost of ten times the CPU time in computation.

A comparison between piecewise linear bases (eqn. 6) and exponential bases (eqn. 7) is also made. One finds that if the thickness of the signal strip $t$ is greater than three times the skin depth $\delta$, the number of linear bases needed is more than for exponential bases to obtain the same accuracy. Because the CPU time is directly proportional to the square of the number of $y$-dependent bases, the use of exponential bases is essential in reducing the computing time at higher frequency. In this study, we chose linear bases when $t<3 \delta$ and exponential bases when $t>3 \delta$.

The effect of varying the strip width $w$ is shown in Fig. 3 in which a comparison of our attenuation constants with the theoretical results of Reference 15 and the measured values of Reference 23 is also included. Good agreement between these results is observed and verifies the accuracy of the new approach. Note that as the strip width increases, the effective dielectric constant increases and the attenuation constant decreases.

Fig. 4 shows the effect of increasing signal strip thick- 


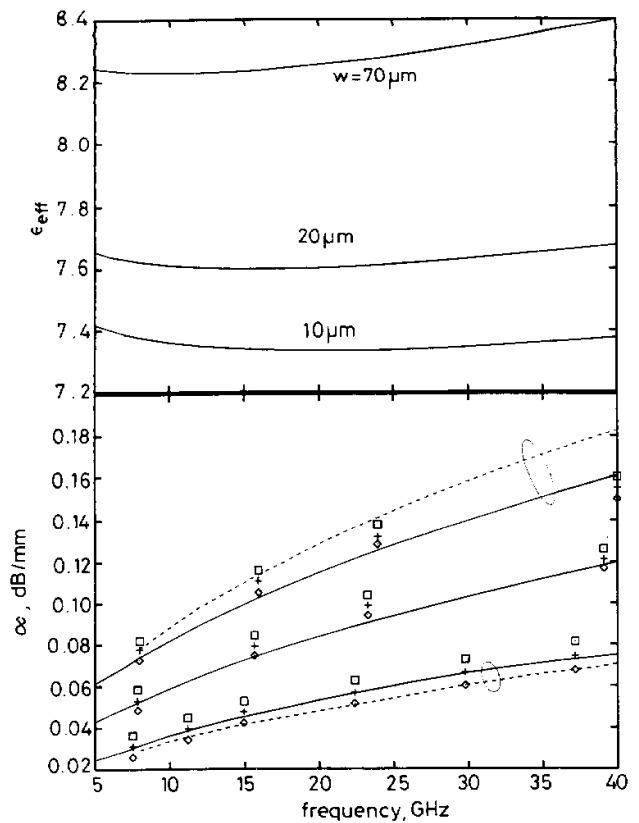

Fig. 3 Effective dielectric constant $\varepsilon_{\text {efs }}$ and attenuation constant $\alpha$ versus frequency with strip width $w$ as parameters. Also included are the results of References 22 and 14 for comparison

$\varepsilon_{r}=12.9$, tan $\delta=3 \times 14^{-4}$, and $h=100 \mu \mathrm{m}$ (also adopted in Figs. 4, 5, 6, and 8). $i=3 \mu \mathrm{m}, b=12 \mu \mathrm{m}, \sigma=\sigma_{b}=4.1 \times 10^{7} \mathrm{~S} / \mathrm{m}$

$\square$ upper limit

[22]

$---[15]$

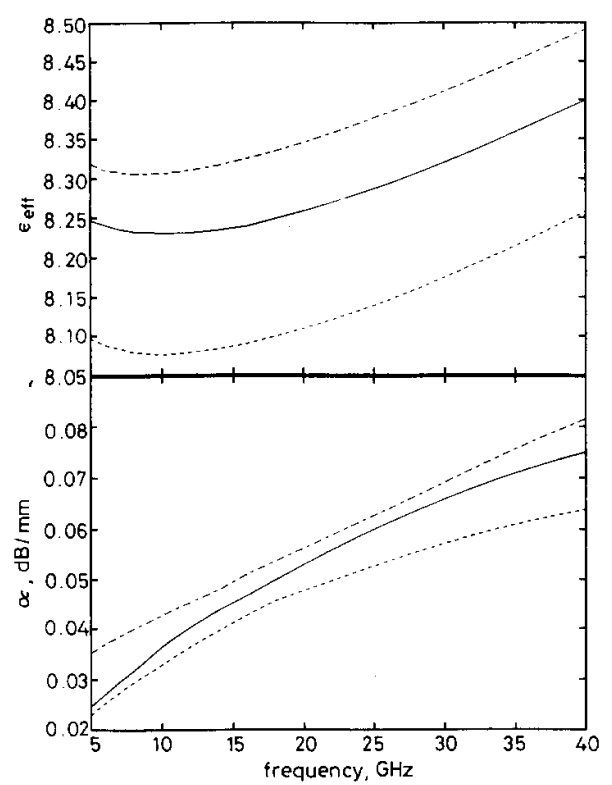

Fig. 4 Effective dielectric constant $\varepsilon_{\text {eff }}$ and attenuation constant $\alpha$ versus frequency with signal strip thickness $t$ as parameters

$\omega=70 \mu \mathrm{m} \quad b=12 \mu \mathrm{m} \quad \sigma=\sigma_{b}=4.1 \times 10^{7} \mathrm{~S} / \mathrm{m}$

-.... $t=1.5 \mu \mathrm{m}$

…... $t=6.0 \mu \mathrm{m}$ ness $t$ on the effective dielectric constant and attenuation constant. As expected, both the effective dielectric constant and attenuation constant decrease as $t$ increases. It is interesting to note that the effective dielectric constant presents a minimum in the neighbourhood of $10 \mathrm{GHz}$. Another point to note is the negative slope of the $\varepsilon_{\text {eff }}$-curve at low frequency. When the conductivity $\sigma$ approaches infinity, current flows only over the strip surface and the effective dielectric constant increases as the frequency increases. However, when the conductivity is finite, the current at low frequency may penetrate deep into the whole strip region, which then introduces an internal inductance to increase the effective dielectric constant at low frequency [23].

The effect of increasing the conductivity $\sigma\left(=\sigma_{b}\right)$ is represented in Fig. 5. Here, both the effective dielectric constant and attenuation constant decrease as $\sigma$ increases.

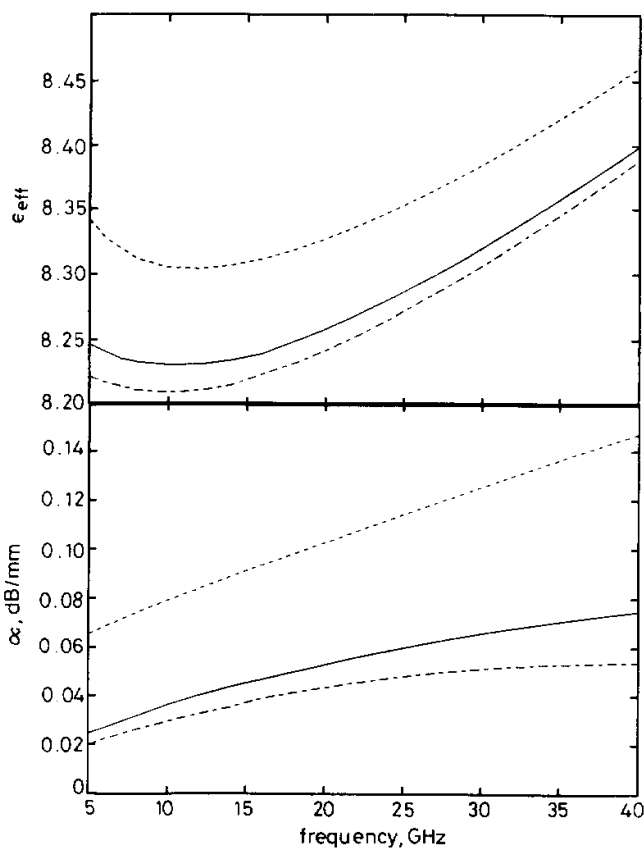

Fig. 5 Effective dielectric constant $\varepsilon_{\text {esf }}$ and attenuation constant versus frequency with conductivities as parameters

$w=70 \mu \mathrm{m} \quad t=3 \mu \mathrm{m} \quad b=12 \mu \mathrm{m}$

- $\sigma=\sigma_{b}=1 \times 10^{7} \mathrm{~s} / \mathrm{m}$

-

The effect of a lossy ground plane is shown in Fig. 6. Also included in this figure are the results for a perfectly conducting (PEC) ground plane $\left(\sigma_{b}=\infty\right)$ for comparison. As expected, the attenuation of the microstrip line with PEC ground plane is less than that with normal conductors. The influence due to the finite conductivity $\sigma_{b}$ of the ground plane is smaller than that due to the conductivity $\sigma$ of the signal strip. The difference in the curves of $\sigma_{b}=\infty$ and $\sigma_{b}=4.1 \times 10^{7} \mathrm{~S} / \mathrm{m}(b=3 \mu \mathrm{m})$ is only $12 \%$ for the attenuation constant and less than $0.3 \%$ for the effective dielectric constant. The effect of ground plane thickness is presented by the curves for $b=3.0 \mu \mathrm{m}$ and $b=1.0 \mu \mathrm{m}$, the difference being less than $2 \%$ for the attenuation constant and less than $0.1 \%$ for the effective dielectric constant.

IEE Proc.-Microw. Antennas Propag., Vol. 142, No. 4, August 1995 
Fig. 7 shows the effect of varying the substrate dielectric constant $\varepsilon_{r}$. As expected, both the effective dielectric constant and attenuation constant increase as $\varepsilon_{r}$

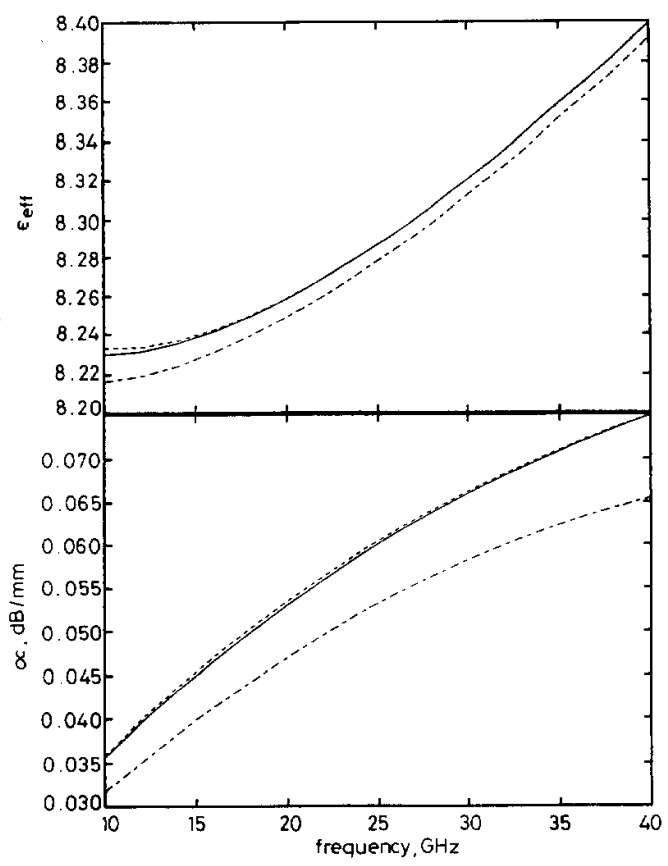

Fig. 6 Effective dielectric constant $\varepsilon_{\text {efs }}$ and attenuation constant $\alpha$ versus frequency with ground plane thickness $b$ and conductivity $\sigma_{b}$ as parameters

$w=70 \mu \mathrm{m} \quad t=3 \mu \mathrm{m} \quad \sigma=4.1 \times 10^{7} \mathrm{~S} / \mathrm{m}$

$$
\begin{aligned}
t=1.0 \mu \mathrm{m} \\
b=3.0 \mu \mathrm{m}
\end{aligned}
$$

$b=3.0 \mu \mathrm{m}$

increases. Also, the attenuation constant is strongly dependent on the substrate dielectric constant. The other phenomenon which should be mentioned is that the higher the dielectric constant, the more the dispersion in the $\varepsilon_{\text {eff }}$-curve.

Shown in Fig. 8 is the distribution of longitudinal current on the signal strip with $w=20 \mu \mathrm{m}$ and frequency, $=7 \mathrm{GHz}$. Because the current converges slowly compared with the propagation constant, we use eight Legendre polynomials and nine piecewise linear functions to get a smooth current distribution. As expected, edge enhancement behaviour is found for the longitudinal current with respect to the $x$ - and $y$ coordinates, but the edge current is finite instead of infinite. To discuss the skin-effect phenomenon due to the finite conductivity, let us examine the curve for $x=0$ in Fig. 8a. From $y=0$ to $y=0.625 t$, the magnitude changes from 1.0 to 0.4 . This behaviour agrees well with the skin depth $\delta$ which is in the order of $1 \mu \mathrm{m}$. It should be noted that the ratio $J_{z}: J_{x}: J_{y}$ ranges roughly from about $1: 10^{-2}: 10^{-4}(10 \mathrm{GHz})$ to $1: 10^{-1}: 10^{-2}(100 \mathrm{GHz})$. Although the transverse currents, $J_{x}$ and $J_{y}$, are much smaller than the longitudinal one $J_{z}$, their existence is important. Without including the transverse currents in the computation, the negative slope of the $\varepsilon_{\text {efr }}$-curve at low frequency would disappear and the attenuation would be less accurate at higher frequencies.
The current distribution around the surface of the signal strip is depicted in Fig. 9. As expected, the edge enhancement effect becomes more clear as frequency increases, which makes the skin depth decrease. Note

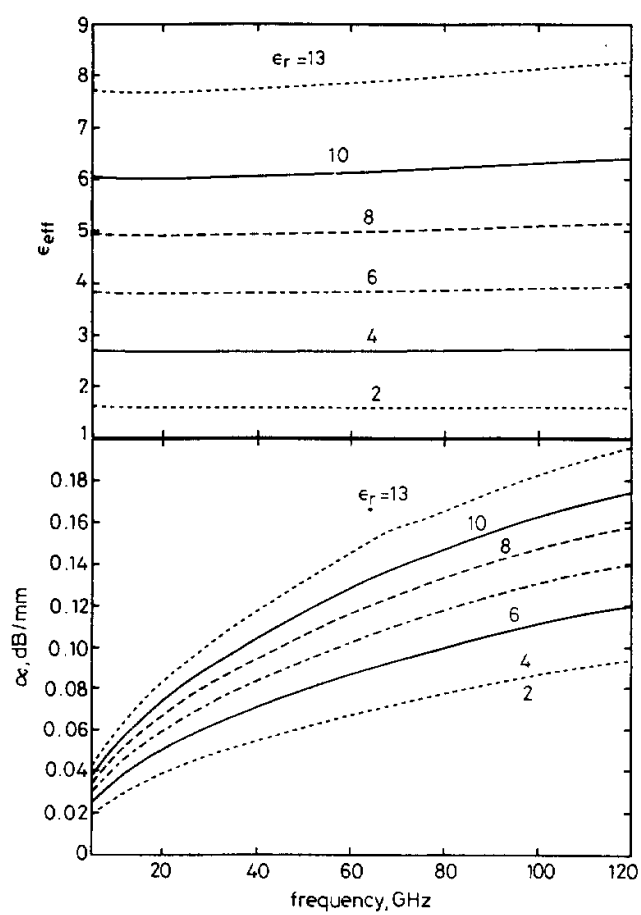

Fig. 7 Effective dielectric constant $\varepsilon_{\text {efs }}$ and attenuation constant $\alpha$ versus frequency with substrate dielectric constant $\varepsilon_{r}$ as parameters

$w=20 \mu \mathrm{m} \quad t=3 \mu \mathrm{m} \quad b=12 \mu \mathrm{m} \quad h=100 \mu \mathrm{m} \quad \tan \delta=0 \quad \sigma=\sigma_{b}=4.1$ $\times 10^{7} \mathrm{~S} / \mathrm{m}$

…… $\varepsilon_{r}=13$

$\varepsilon_{r}=10$

$\varepsilon_{r}=8$

$\varepsilon_{r}=6$

$\varepsilon_{r}=4$

$\varepsilon_{r}=2$

also that as frequency increases the current on the top side $(y=t)$ decreases.

\section{Conclusions}

In this study, a modified spectral-domain approach has been proposed to deal with microstrip lines with a layer structure in which both the thickness and conductivity of the signal strip and ground plane are finite. The effective dielectric constant and attenuation constant of this lossy microstrip line have been discussed in detail, together with the longitudinal current distributions along the signal strip.

The proposed approach can be applied to structures with superconductor signal strip and/or semiconductor substrates. In addition, extension to coplanar strips and coplanar waveguides is straightforward.

In this work, two kinds of $y$-dependent bases (piecewise linear and exponential) are proposed for the unknown strip current. The disadvantage of the piecewise linear bases is that the number of $y$-dependent bases used is proportional to the thickness-to-skin depth ratio $t / \delta$. If 
the value $t / \delta$ is larger than three, the computation cost of using linear bases is expensive. To avoid the increased CPU time as $t / \delta$ increases, exponential $y$-dependent bases should be used.
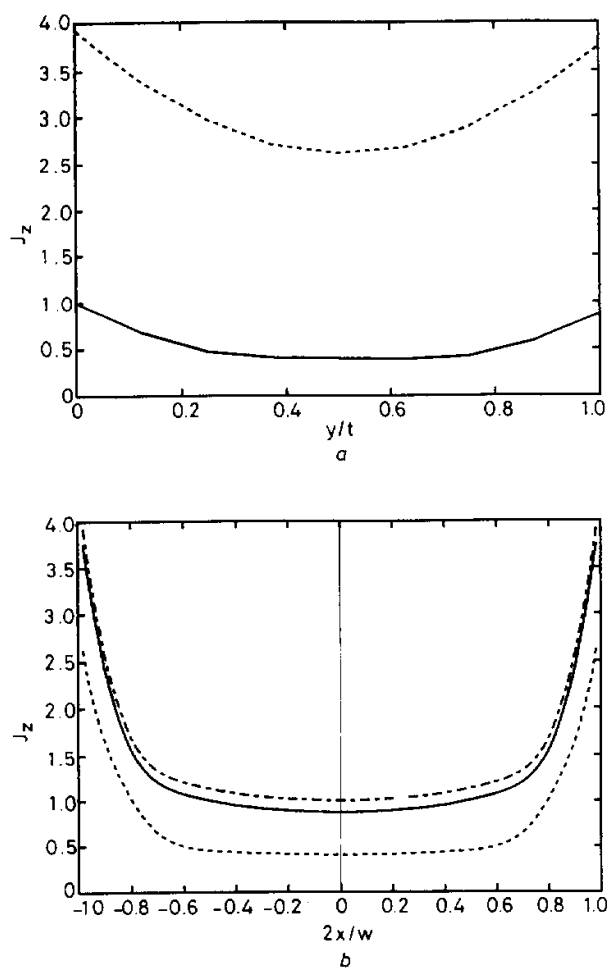

Fig. 8 Longitudinal current distributions

\section{a $y$-direction}

$b \quad x$-direction
frequency $=7 \mathrm{GHz} \quad w=20 \mu \mathrm{m} \quad \mathrm{t}=3 \mu \mathrm{m} \quad b=12 \mu \mathrm{m} \quad a=\sigma_{\mathrm{b}}=4.1 \times 10^{7} \mathrm{~S} / \mathrm{m}$

a $---x=w / 2$

$\begin{aligned} x & =0 \\ y & =0\end{aligned}$

$\longrightarrow \begin{aligned} & y \\ & y=t / 2\end{aligned}$

$-\ldots y=t$

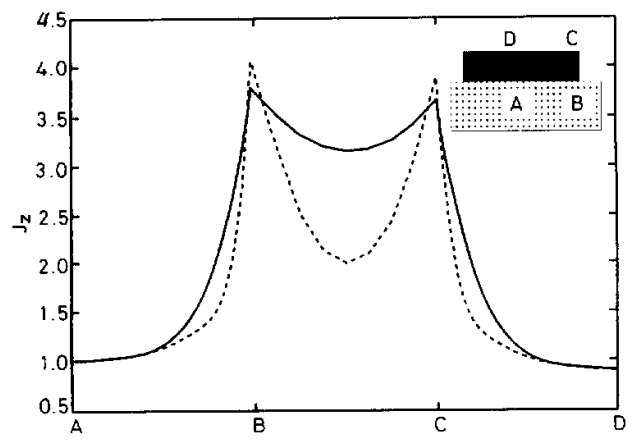

Fig. 9 Longitudinal current distributions along the surface of the signal strip

$w=20 \mu \mathrm{m} \quad t=3 \mu \mathrm{m} \quad b=12 \mu \mathrm{m} \quad \sigma=\sigma_{b}=4.1 \times 10^{7} \mathrm{~S} / \mathrm{m}$

$\begin{aligned}---f & =3 \mathrm{GHz}\end{aligned}$

362

\section{References}

1 HEINRICH, W.: 'Conductor loss on transmission lines in monolithic microwave and millimeter-wave integrated', Int. J. Microwave Millimeter-Wave Computer-Aided Engng, 1992, 2, (3), pp. 155-167

2 SHIH, C., WU, R.B., JENG, S.K., and CHEN, C.H.: 'Frequencydependent characteristics of open microstrip lines with finite strip thickness', IEEE Trans. Microwave Theory Technol., 1989, 37, (4), pp. 793-795

3 KITAZAWA, T.: 'Metallization thickness effect of striplines with anisotropic media: quasistatic and hybrid-mode analysis', IEEE Trans. Microwave Theory Tech., 1989, 37, (4), pp. 769-775

4 ZHU, L., and YAMASHITA, E.: 'New method for the analysis of dispersion characteristics of various planar transmission lines with finite metallization thickness', IEEE Trans. Microwave and Guided Wave Lett., 1991, 1, (7), pp. 164-166

5 ZHU, L., and YAMASHITA, E.: 'Full-wave boundary integral equation method for suspended planar transmission lines with pedestals and finite metallization thickness', IEEE Trans. Microwave Theory Technol., 1993, 41, (3), pp. 478-483

6 KOLLIPARA, R.T., and TRIPATHI, V.K.: 'Dispersion characteristic of moderately thickness microstrip lines by the spectral domain method', IEEE Trans. Microwave and Guided Wave Lett., 1992, 2, (3), pp. 100-101

7 COLLIN, R.E.: 'Field theory of guided waves'. 2nd edn. (IEEE Press, New York, 1991)

8 PUCEL, R.A., MASSÉ, D.J., and HARTWIG, C.P.: 'Losses in microstrip', IEEE Trans. Microwave Theory Technol., 1968, 16, (6), pp. $342-350$

9 HARRINGTON, R.F., and WEI, C.: 'Losses on multiconductor transmission lines in multilayered dielectric media', IEEE Trans. Microwave Theory Technol., 1984, 32, (7), pp. 705-710

10 BARSOTTI, E.L, KUESTER, E.F, and DUNN, J.M.: 'A simple method to account for edge shape in the conductor loss in microstrip', IEEE Trans. Microwave Theory Technol, 1991, 39, (1), Pp. 98-106

11 SYAHKAL, D.M., and DAVIES, J.B.: 'Accurate solution of microstrip and coplanar structures for dispersion and for dielectric and conductor losses", IEEE Trans. Microwave Theory Technol., 1979, 27, (7), pp. 694-699

12 ALESSANDRI, $F$, BAINI, $G$, D'INZEO, $G$, and SORRENTINO, R. ' Conductor loss computation in multiconductor MIC's by transverse resonance technique and modified perturbational method', IEEE Trans. Microwave and Guided Wave Lett., 1992, 2, (6), pp. 250-252

13 KITAZAWA, T., and ITOH, T.: 'Propagation characteristics of coplanar-type transmission lines with lossy media', IEEE Trans. Microwave Theory Technol., 1991, 39, (10), pp. 1694-1700

14 KITAZAWA T, POLIFKO, D, and OGAWA, H.: 'Analysis of CPW for LiNb optical modulator by extended spectral domain approach', IEEE Microwave Guided Wave Lett., 1992, 2, (8), pp. 313-315

15 KITAZAWA, T.: 'Loss calculation of single and coupled strip lines by extended spectral domain approach', IEEE Microwave Guided Wave Lett.. 1993, 3, (7), pp. 211-213

16 POND, JM. KROWNE, C.M, and CARTER, W.L.: On the application of complex resistive boundary conditions to model transmission lines consisting of very thin superconductors', IEEE Trans. Microwave Theory Technol., 1989, 37, (1), pp. 181-190

$17 \mathrm{KUO}, \mathrm{C.W}$., and ITOH, T.: 'A flexible approach combining the spectral domain method and impedance boundary condition for the analysis of microstrip lines', IEEE Trans. Microwave and Guided Wave Letr. 1991, 1, (7), pp. 172-174

18 NGHIEM, D. WILLIAMS, J.T, and JACKSON, D.R.: 'A general analysis of propagation along multiple-layer superconducting stripline and microstrip transmission lines', IEEE Trans. Microwave Theory Technol., 1991, 39, (9), pp. 1553-1565

19 VAN DEVENTER, T.E., KATEHI, P.B., and CANGELLARIS A.C.: 'An integral equation method for the evaluation of conductor and dielectric losses in high-frequency interconnects', IEEE Trans. Microwave Theory Technol., 1989, 37, (12), pp. 1964-1972

20 HEINRICH, W.: 'Full-wave analysis of conductor losses on MMIC transmission lines', IEEE Trans. Microwave Theory Technol., 1990, 38, (10), pp. 1468-1472

21 SCHMUCKLE, F.J., and PREGLA, R.: 'The method of lines for the analysis of lossy planar waveguides', IEEE Trans. Microwave Theory Technol., 1990, 38, (10), pp. 1473-1479

22 KIANG, J.F.: 'Integral equation solution to the skin effect problem in conductor strips of finite thickness', IEEE Trans. Microwave Theory Technol., 1991, 39, (3), pp. 452-460

23 GOLDFARB, M., and PLATZKER, A.: 'Losses in GaAs microstrip', IEEE Trans. Microwave Theory Technol., 1990, 38, (12), pp 1957-1963

IEE Proc.-Microw. Antennas Propag., Vol. 142, No. 4, August 1995 
24 SCHROEDER, W., and WOLFF, I.: Full-wave loss analysis of normal and superconducting transmission lines by hybrid-mode boundary integral equation method', 1991 IEEE MTT-S Dig., I, pp. 341-344

25 ITOH, T: 'Numerical techniques for microwave and millimeterwave passive structures' (John Wiley, New York, 1989)

26 DAS, N.K., and POZAR, D.M.: 'A generalized spectral-domain Green's function for multilayer dielectric substrates with application to multilayer transmission lines', IEEE Trans. Microwave Theory Technol., 1987, 36, pp. 326-335

\section{Appendix}

The spectral-domain dyadic Green functions $\tilde{G}_{z z}, \tilde{G}_{z x}$, $\tilde{G}_{x z}, \tilde{G}_{x x}, \tilde{G}_{y z}$, and $\tilde{G}_{y x}$ for the layer structure Fig. $1 b$ can be developed by the method of References 25 and 26 . Then, the Green functions $\tilde{G}_{z y}$ and $\tilde{G}_{x y}$ can be derived from $\bar{G}_{y z}$ and $\bar{G}_{y x}$ by reciprocity, and $G_{y y}$ can be found easily from $\tilde{G}_{z y}$ and $\tilde{G}_{x y}$. Included here is a typical one such as

$$
\begin{aligned}
\tilde{G}_{z z}= & \frac{-1}{2 \omega \varepsilon_{0}\left(k_{x}^{2}+k_{z}^{2}\right)}\left[k_{z}^{2} \beta_{0}\left(e^{-j \beta_{0}\left|y-y^{\prime}\right|}-\Gamma_{A 0}^{\prime} e^{-j \beta_{0}\left(y+y^{\prime}\right)}\right)\right. \\
& \left.+\frac{k_{x}^{2} k_{0}^{2}}{\beta_{0}}\left(e^{-j \beta_{0}\left|y-y^{\prime}\right|}+\Gamma_{F 0}^{\prime} e^{-j \beta_{0}\left(y+y^{\prime}\right.}\right)\right]
\end{aligned}
$$

$$
\begin{gathered}
\frac{\beta_{1}}{\varepsilon_{r}}\left(\frac{\beta_{2}}{\varepsilon_{r 2}}+j \frac{\beta_{0}}{\varepsilon_{0}} \tan \beta_{2} b\right) \\
\Gamma_{A 1}=e^{-2 j \beta_{1} h} \frac{-\frac{\beta_{2}}{\varepsilon_{r 2}}\left(\frac{\beta_{0}}{\varepsilon_{0}}+j \frac{\beta_{2}}{\varepsilon_{r 2}} \tan \beta_{2} b\right)}{\frac{\beta_{1}}{\varepsilon_{r}}\left(\frac{\beta_{2}}{\varepsilon_{r 2}}+j \frac{\beta_{0}}{\varepsilon_{0}} \tan \beta_{2} b\right)} \\
+\frac{\beta_{2}}{\varepsilon_{r 2}}\left(\frac{\beta_{0}}{\varepsilon_{0}}+j \frac{\beta_{2}}{\varepsilon_{r 2}} \tan \beta_{2} b\right)
\end{gathered}
$$

$\beta_{1}\left(\beta_{2}+j \beta_{0} \tan \beta_{2} b\right)$

$\Gamma_{F 1}=e^{-2 j \beta_{1} h} \frac{-\beta_{2}\left(\beta_{0}+j \beta_{2} \tan \beta_{2} b\right)}{\beta_{1}\left(\beta_{2}+j \beta_{0} \tan \beta_{2} b\right)}$

$$
+\beta_{2}\left(\beta_{0}+j \beta_{2} \tan \beta_{2} b\right)
$$

and $\beta_{i}^{2}=k_{i}^{2}-k_{x}^{2}-k_{z}^{2}(i=0,1,2), k_{0}^{2}=\omega^{2} \varepsilon_{0} \mu_{0}, k_{1}^{2}=$ $\omega^{2} \varepsilon_{0} \mu_{0} \varepsilon_{r}(1-j \tan \delta), k_{2}^{2}=-j \omega \sigma_{b}$. It should be emphasised again that the ground plane is now regarded as a lossy layer with wavenumber $k_{2}$ and thickness $b$ therefore the effect of lossy ground may be included and discussed by these Green's functions.

where

$$
\begin{aligned}
& \Gamma_{A 0}^{\prime}=\left(\beta_{0}-Z_{A 1}\right) /\left(\beta_{0}+Z_{A 1}\right) \\
& \Gamma_{F 0}^{\prime}=\left(\beta_{0}-Z_{F 1}\right) /\left(\beta_{0}+Z_{F 1}\right) \\
& Z_{A 1}=\frac{\beta_{1}}{\varepsilon_{P}(1-j \tan \delta)}\left(1-\Gamma_{A 1}\right) /\left(1+\Gamma_{A 1}\right) \\
& Z_{F 1}=\beta_{1}\left(1-\Gamma_{F 1}\right) /\left(1+\Gamma_{F 1}\right)
\end{aligned}
$$

